Artículo

\title{
Uso de Opstat para validar resultados en un dialélico parcial con ocho líneas de maíz evaluadas en un ambiente
}

\author{
Gerardo Jasso-Bobadilla ${ }^{1 \S}$ \\ Andrés González-Huerta ${ }^{2}$ \\ Delfina de Jesús Pérez-López ${ }^{2}$ \\ J. Ramón Pascual Franco-Martínez ${ }^{2}$ \\ Martín Rubí-Arriaga ${ }^{2}$ \\ Jaime Mejía-Carranza ${ }^{3}$ \\ ${ }^{1}$ Programa de Doctorado en Ciencias Agropecuarias y Recursos Naturales-Universidad Autónoma del \\ Estado de México-Instituto de Ciencias Agropecuarias y Rurales-Campus Universitario 'El Cerrillo'. El \\ Cerrillo Piedras Blancas, Toluca, Estado de México, México. Tel. 722 2965552, ext. 117. \\ (pcarn@uaemex.mx). ${ }^{2}$ Centro de Investigación y Estudios Avanzados en Fitomejoramiento-Facultad de \\ Ciencias Agrícolas. AP. 435. Tel. 722 2965518, ext. 148. (agonzalezh@uaemex.mx; djperezl@uaemex.mx; \\ jrfrancom@uaemex.mx; mrubia@uaemex.mx). ${ }^{3}$ Centro Universitario Tenancingo. Carretera Tenancingo- \\ Villa Guerrero km 1.5, Tenancingo, Estado de México, México. CP. 52400. Tel. 7141407724. \\ (jmejiac@uaemex.mx).
}

Autor para correspondencia: gjabo75@gmail.com.

\section{Resumen}

Se describen los procedimientos de muestreo en un experimento dialélico parcial aplicando el método de Kempthorne y Curnow (1961) a ocho líneas de maíz $\left(\mathrm{S}_{7}\right)$ derivadas de la misma variedad, utilizadas cada una en cinco cruzamientos ( $s=5$ ). Para los 20 híbridos de cruza simple se indican los cálculos para obtener el análisis de varianza para un ambiente con un diseño experimental de bloques completos al azar con cuatro repeticiones. Los efectos entre cruzas se dividen en aptitud combinatoria general y específica utilizando álgebra de matrices, se estiman los efectos de $\mathrm{g}_{\mathrm{i}} \mathrm{y} \mathrm{S}_{\mathrm{ij}}$, para cada progenitor y en cada cruza, respectivamente. Además, se calculan los componentes de varianza y las heredabilidades en sentido amplio y estrecho. En todas las etapas se indica como verificar los cálculos empleando una calculadora de escritorio y, finalmente, se validan los resultados usando el paquete estadístico Opstat.

Palabras clave: aptitud combinatoria, dialélico incompleto, heredabilidad, matriz circulante.

Recibido: octubre de 2021

Aceptado: enero de 2022 


\section{Introducción}

En un experimento de cruzas dialélicas completo hay $\mathrm{p}^{2}$ combinaciones genéticas que incluyen $\mathrm{p}$ progenitores $(i=j), p(p-1) / 2$ cruzas directas $(C D ; i<j)), y p(p-1) / 2$ cruzas recíprocas $(C R ; i>j)$ (Griffing, 1956). Este diseño ha sido ampliamente utilizado en fitomejoramiento (Saavedra et al., 2021) pero es impráctico cuando $\mathrm{p}$ aumenta debido a que CD y CR también se incrementan; una situación similar se presentó cuando se diseñaron experimentos $2^{\mathrm{H}}$, con $\mathrm{H}$ variando de 2 a 8 , para ser analizados con InfoStat e InfoGen, si H= 8 habrá 256 tratamientos y 247 interacciones. Para ahorrar tiempo y recursos podría diseñarse un factorial fraccionado que incluya sólo a un subconjunto de éstos (Pérez et al., 2021). Si 13 progenitores, 78 CD y 78 CR serán estudiados, aun para los diseños en látice rectangular habría serias limitantes (González et al., 2007).

Cochran y Cox (1958); Gomez y Gomez (1984); Martínez (1998) mostraron los planes básicos para látice balanceado y parcialmente balanceado; se podrían aleatorizar hasta 144 tratamientos en un látice triple 12 x 12. Si en un ambiente se evalúa maíz (Zea mays L.) en tres repeticiones con parcelas de tres surcos de $5 \mathrm{~m}$ de longitud, cada uno distanciado a $0.8 \mathrm{~m}$, para evaluar esos 144 tratamientos serían necesarios casi $6400 \mathrm{~m}^{2}$ (repeticiones separadas a $2 \mathrm{~m}$ y bloques incompletos distanciados a $1.5 \mathrm{~m}$ ). Este problema será mayor al considerar varios años, localidades o combinaciones de éstos. En el otro extremo, con $\mathrm{p}=4$, las estimaciones de aptitud combinatoria general serán sesgadas debido al pequeño tamaño de muestra utilizando.

Ambas limitantes han conducido al muestreo de las cruzas incluyendo más progenitores sin afectar la eficiencia de la técnica dialélica. Hinkelmann y Stern (1960); Kempthorne y Curnow (1961); Fyfe y Gilbert (1963); Rojas (1973) diseñaron metodologías para el muestreo y análisis de un dialélico parcial. Kempthorne y Curnow (1961) inventaron una técnica dialélica incompleta a partir del método 4 de Griffing (1956) usando una muestra de tamaño ps/2, dónde s es el número de veces que cada progenitor (p) es utilizado en los cruzamientos; $\mathrm{p}$ y s no pueden ser simultáneamente números nones pero la precisión con que se estiman los parámetros de interés para el fitomejorador depende de ambos valores. Murthy et al. (1966); Chaudhary et al. (1977) precisaron que el sesgo es mayor cuando s es menor que $\mathrm{p} / 2$. Con $\mathrm{s}=\mathrm{p}-1$ se tendrá el método 4 de Griffing (1956).

No obstante, deberá considerarse que el diseño experimental de bloques completos al azar podría perder eficiencia si p y s se incrementan significativamente debido a que el tamaño del bloque aumentará y habrá menor uniformidad dentro de éste por lo que el error experimental será mayor así, una opción muy deseable sería dividir cada repetición en bloques incompletos balanceados. Este ha sido estudiado por Shunmigathai y Srinivasan (2012), quienes han discutido la disminución de la eficiencia de un dialélico incompleto, incluso con la pérdida de una o más observaciones.

La cruza dialélica parcial se ha utilizado principalmente para estimar parámetros genéticos en híbridos de cruza simple formados con líneas endogámicas derivadas de la misma población evaluadas en un diseño experimental de bloques completos al azar, bajo la consideración de un modelo genético de efectos fijos (Christie y Shattuck, 1992; Mumtaz et al., 2015; Awata et al., 2018), pero Miranda y Vencousky (1999) y Silva et al. (2017) entre otros, han desarrollado otra metodología para analizar un dialélico parcial formado con dos grupos de líneas o progenitores genéticamente contrastantes; ellos realizaron cruzamientos entre material exótico y genotipos adaptados localmente. 


\section{Diseño de las cruzas}

Primero deberá calcularse $\mathrm{k}$, que debe ser un número entero. Así: $\mathrm{k}=\frac{(\mathrm{p}+1-\mathrm{s})}{2}$. Los progenitores, aleatorizados y numerados consecutivamente, generarán las cruzas: Progenitor 1 x progenitor $\mathrm{k}+1$, $\mathrm{k}+2, \mathrm{k}+3, \ldots, \mathrm{k}+\mathrm{s}$. Progenitor $2 \mathrm{x}$ progenitor $\mathrm{k}+2, \mathrm{k}+3, \mathrm{k}+4, \ldots, \mathrm{k}+1+\mathrm{s}, \ldots$, Progenitor $\mathrm{p}$ $\mathrm{x}$ progenitor $\mathrm{k}+\mathrm{p}, \mathrm{k}+\mathrm{p}+1, \ldots, \mathrm{k}+\mathrm{p}-1+\mathrm{s}$. Con ocho líneas de maíz, $\mathrm{s}=5 \mathrm{y} \mathrm{k}=2$, las 20 cruzas que serán muestreadas son: Línea 1 x líneas $2+1,2+2,2+3,2+4,2+5=1 \times 3,1 \times 4,1 \times 5,1 \times 6,1 \times 7$. Línea 2 x líneas $2+2,2+3,2+4,2+5,2+6=2 \times 4,2 \times 5,2 \times 6,2 \times 7,2 \times 8$. Línea 3 x líneas 2+3, 2+4, $2+5,2+6,2+7=3 \times 5,3 \times 6,3 \times 7,3 \times 8$. Línea $4 \times$ líneas $2+4,2+5,2+6,2+7,2+8=4 \times 6,4 \times 7,4 \times 8$. Línea $5 \times$ líneas $2+5,2+6,2+7,2+8,2+9=5 \times 7,5 \times 8$. Línea $6 \times$ líneas $2+6,2+7,2+8,2+9,2+10=$ 6x8. La línea 7 se cruzaría con los machos $2+7,2+8,2+9,2+10$ y $2+11$, como los machos son mayores que p se aplica múltiplos de $8: 7 x(9-8)=7 \times 1 ; 7 x(10-8)=7 \times 2 ; 7 x(11-8)=7 \times 3 ; 7 x(12-8)=$ $7 \times 4 ; 7 x(13-8)=7 x 5$, pero se eliminan por ser CR y también las cruzas con la línea 8 (Cuadro 1).

Cuadro 1. 20 cruzas muestreadas con el método de Kempthorne y Curnow (1961).

\begin{tabular}{|c|c|c|c|c|c|c|c|c|}
\hline \multirow{2}{*}{ Línea i $(+$ ( ) } & \multicolumn{8}{|c|}{ Línea j (ठ઼) } \\
\hline & $\mathrm{P} 1$ & $\mathrm{P} 2$ & P3 & $\mathrm{P} 4$ & P5 & P6 & P7 & P8 \\
\hline $\mathrm{P} 1$ & 0 & 0 & 1 & 1 & 1 & 1 & 1 & 0 \\
\hline $\mathrm{P} 2$ & 0 & 0 & 0 & 1 & 1 & 1 & 1 & 1 \\
\hline P3 & 1 & 0 & 0 & 0 & 1 & 1 & 1 & 1 \\
\hline $\mathrm{P} 4$ & 1 & 1 & 0 & 0 & 0 & 1 & 1 & 1 \\
\hline P5 & 1 & 1 & 1 & 0 & 0 & 0 & 1 & 1 \\
\hline P6 & 1 & 1 & 1 & 1 & 0 & 0 & 0 & 1 \\
\hline $\mathrm{P} 7$ & 1 & 1 & 1 & 1 & 1 & 0 & 0 & 0 \\
\hline P8 & 0 & 1 & 1 & 1 & 1 & 1 & 0 & 0 \\
\hline
\end{tabular}

$a_{\mathrm{ij}}=1$ o $a_{\mathrm{ij}}=0$ si la cruza ixj es o no muestreada, respectivamente. También se indican CR.

\section{Análisis de varianza (Anava) general}

El modelo estadístico para un diseño experimental de bloques completos al azar es: $Y_{\mathrm{ij}}=\mu+\tau_{\mathrm{i}}+\beta_{\mathrm{j}}$ $+\varepsilon_{\mathrm{ij}}$. Dónde: $\mu$ es la media aritmética de los tr datos, $\tau_{\mathrm{i}}$ es el efecto del i-ésimo tratamiento, $\beta_{\mathrm{j}}$ es la contribución de la j-ésima repetición, y $\varepsilon_{\mathrm{ij}}$ es el error experimental o residual del modelo.

\section{Etapas para obtener un Anava general}

E1. Concentrar los datos en una tabla: las hileras (subíndices ij) representarán cruzas y las columnas (subíndice k) repeticiones, calcular totales y medias aritméticas (Cuadro 2).

Cuadro 2. Rendimiento de grano $\left(\mathrm{t} \mathrm{ha}^{-1}\right)$ de 20 híbridos de cruza simple de maíz.

\begin{tabular}{ccccccc}
\hline \multirow{2}{*}{ Cruza (ij) } & \multicolumn{7}{c}{ Repeticiones (k) } \\
\cline { 2 - 7 } & R1 & R2 & R3 & R4 & Total & Media \\
\hline $1 \times 3$ & 7.56 & 4.5 & 8.68 & 8.43 & 29.17 & 7.292 \\
$1 \times 4$ & 6.62 & 7.33 & 6.87 & 7.16 & 27.98 & 6.995 \\
$1 \times 5$ & 7.1 & 6.87 & 6.7 & 5.83 & 26.5 & 6.625 \\
\hline
\end{tabular}




\begin{tabular}{ccccccc}
\hline \multirow{2}{*}{ Cruza (ij) } & \multicolumn{5}{c}{ Repeticiones (k) } \\
\cline { 2 - 6 } $1 \times 6$ & $\mathrm{R} 1$ & $\mathrm{R} 2$ & $\mathrm{R} 3$ & $\mathrm{R} 4$ & Total & Media \\
\hline $1 \times 7$ & 7.36 & 4.81 & 7.64 & 7.64 & 27.45 & 6.862 \\
$2 \times 4$ & 6.06 & 6.68 & 5.2 & 6.37 & 24.31 & 6.077 \\
$2 \times 5$ & 6.35 & 6.16 & 6.56 & 6.66 & 25.73 & 6.432 \\
$2 \times 6$ & 7.58 & 5.33 & 7.85 & 7.41 & 28.17 & 7.042 \\
$2 \times 7$ & 8.66 & 7.29 & 8.35 & 6.75 & 31.05 & 7.762 \\
$2 \times 8$ & 6.91 & 6.62 & 7.02 & 7.12 & 27.67 & 6.917 \\
$3 \times 5$ & 7.62 & 9.41 & 6.99 & 7.41 & 31.43 & 7.857 \\
$3 \times 6$ & 6.99 & 5.7 & 6.27 & 6.75 & 25.71 & 6.427 \\
$3 \times 7$ & 6.62 & 5.23 & 9.16 & 7.95 & 28.96 & 7.24 \\
$3 \times 8$ & 6.27 & 5.83 & 6.62 & 6.6 & 25.32 & 6.33 \\
$4 \times 6$ & 6.87 & 5.12 & 7.56 & 7.73 & 27.28 & 6.82 \\
$4 \times 7$ & 5.73 & 4.37 & 5.2 & 8.08 & 23.38 & 5.845 \\
$4 \times 8$ & 6.95 & 6.66 & 6.45 & 6.08 & 26.14 & 6.535 \\
$5 \times 7$ & 7.7 & 5.31 & 7.08 & 8.33 & 28.42 & 7.105 \\
$5 \times 8$ & 6.18 & 5.62 & 6.14 & 5.83 & 23.77 & 5.942 \\
$6 \times 8$ & 6.89 & 5.75 & 7.89 & 8.08 & 28.61 & 7.152 \\
Total & 5.77 & 5.68 & 8.2 & 6.83 & 26.48 & 6.62 \\
& 137.79 & 120.27 & 142.43 & 143.04 & 543.53 & 135.877 \\
\hline
\end{tabular}

E2. Definir el formato del Anava general (Cuadro 3). Concentrar los cálculos anteriores en el formato del Anava general

Cuadro 3. Anava general para rendimiento de grano.

\begin{tabular}{ccccccc}
\hline \multirow{2}{*}{$\begin{array}{c}\text { Fuente de } \\
\text { variación }\end{array}$} & Grados de & Suma de & Cuadrados & & \multicolumn{2}{c}{ Valores de F } \\
\cline { 5 - 7 } & libertad & cuadrados & medios & & Calculado & \multicolumn{2}{c}{ Tablas } \\
\hline Repeticiones & 3 & 17.07 & 5.69 & $6.84^{* *}$ & 2.78 & 4.16 \\
Cruzas & 19 & 22.43 & 1.18 & $1.42 \mathrm{~ns}$ & 1.75 & 2.22 \\
Error & 57 & 47.38 & 0.83 & & & \\
Total & 79 & 86.89 & & & & \\
\hline
\end{tabular}

${ }^{* *}=$ altamente significativo $(p=0.01) ; \mathrm{ns}=$ no significativo $(p=0.05) ; \mathrm{CV}=13.4 \%$.

\section{Calcular grados de libertad (GL)}

En esta sección, $\mathrm{t}$ es el número de cruzas muestreadas, por lo que $\mathrm{t}=\mathrm{ps} / 2$. GL del total $=(\mathrm{ps} / 2)(\mathrm{r})$ $1=20(4)-1=79$; GL rep= r - 1= 4 - 1=3; GL cruzas= (ps/2) $-1=20-1=19$; GL del error $=[(\mathrm{ps} / 2)-$ 1] $(\mathrm{r}-1)=\mathrm{GL}$ total $-\mathrm{GL}$ rep $-\mathrm{GL}$ cruzas $=57$. 


\section{Estimar sumas de cuadrados (SC)}

Aquí se introduce la restricción $\mathrm{i}<\mathrm{j}$. Adicionalmente, $\mathrm{ij} \neq 12,18,23,34,45,56,67$ y 78, son las cruzas no muestreadas en el dialélico parcial, en orden de hembra y macho. SC total= $\sum_{\mathrm{i}=1}^{\mathrm{p}} \sum_{\mathrm{j}=1}^{\mathrm{p}} \sum_{\mathrm{k}=1}^{\mathrm{r}} \mathrm{Y}_{\mathrm{ijk}}^{2}-\frac{\mathrm{Y}_{\ldots i}^{2}}{\frac{\mathrm{psr}}{2}}=\left(7.56^{2}+6.62^{2}+,+6.83^{2}\right)-\left[(543.53)^{2} / 80\right]=86.9 . \mathrm{SC}$ rep $=\frac{\sum_{\mathrm{k}=1}^{\mathrm{r}} \mathrm{Y}_{. \mathrm{k}}^{2}}{\left(\frac{\mathrm{p}}{2}\right)}-\frac{\mathrm{Y}_{\ldots .}^{2}}{\left(\frac{\mathrm{psr}}{2}\right)}=$ $\frac{137.79^{2}+, \ldots, 143.04^{2}}{20}-\frac{543.53^{2}}{20(4)}=17.07 . \mathrm{SC}$ cruzas $=\frac{\sum_{\mathrm{i}=1}^{\mathrm{p}} \sum_{\mathrm{j}=1}^{\mathrm{p}} \mathrm{Y}_{\mathrm{ij} .}^{2}}{\mathrm{r}}-\frac{\mathrm{Y}_{\ldots .}^{2}}{\left(\frac{\mathrm{pSr}}{2}\right)}=\frac{29.17^{2}+27.98^{2}+26.50^{2}+, \ldots,+26.48^{2}}{4}-\frac{543.53^{2}}{20(4)}=$ 22.439. SC error $=\mathrm{SC}$ total-SC rep-SC cruzas $=86.898-17.07-22.439=47.384$.

\section{Determinar cuadrados medios $(\mathrm{CM})$}

$\mathrm{CM}$ rep= SC rep/r -1= 17.07/3= 5.691; CM cruzas= SC cruzas $/[(\mathrm{ps} / 2)-1]=22.439 / 19=1.181 ; \mathrm{CM}$ error $=\mathrm{SC}$ error/(r-1) $[(\mathrm{ps} / 2)-1]=47.384 / 57=0.8312$.

\section{Obtener valores de $\mathbf{F}$ calculados}

$\mathrm{F}$ rep $=\mathrm{CM}$ rep/CM error= 5.691/0.8312=6.846. $\mathrm{F}$ trat $=\mathrm{CM}$ cruzas $/ \mathrm{CM}$ error=1.181/0.8312=1.42.

Definición de la matriz circulante $\underline{A}=[s I+N]$

$$
\begin{aligned}
& \underline{\mathrm{A}} 8 \times 8=\left[\begin{array}{cccccccc}
5 & 0 & 1 & 1 & 1 & 1 & 1 & 0 \\
0 & 5 & 0 & 1 & 1 & 1 & 1 & 1 \\
1 & 0 & 5 & 0 & 1 & 1 & 1 & 1 \\
1 & 1 & 0 & 5 & 0 & 1 & 1 & 1 \\
1 & 1 & 1 & 0 & 5 & 0 & 1 & 1 \\
1 & 1 & 1 & 1 & 0 & 5 & 0 & 1 \\
1 & 1 & 1 & 1 & 1 & 0 & 5 & 0 \\
0 & 1 & 1 & 1 & 1 & 1 & 0 & 5
\end{array}\right] \text { La suma en cada hilera }(\mathrm{r}) \text { o columna }(\mathrm{c}) \text { es: } \\
& \sum_{\mathrm{r}=0}^{\mathrm{p}-1} \mathrm{a}_{\mathrm{r}}=\sum_{\mathrm{c}=0}^{\mathrm{p}-1} \mathrm{a}_{\mathrm{c}}=2 \mathrm{~s}=2(5)=10
\end{aligned}
$$

La inversa de $\underline{\mathrm{A}}$ se obtuvo con una calculadora de matrices (https://matrixcalc.org.es). Así:

$$
\underline{\mathrm{A}}^{-1} 8 \times 8=\left[\begin{array}{cccccccc}
0.238 & 0.027 & -0.029 & -0.044 & -0.047 & -0.044 & -0.029 & 0.027 \\
0.027 & 0.238 & 0.027 & -0.029 & -0.044 & -0.047 & -0.044 & -0.029 \\
-0.029 & 0.027 & 0.238 & 0.027 & -0.029 & -0.044 & -0.047 & -0.044 \\
-0.044 & -0.029 & 0.027 & 0.238 & 0.027 & -0.029 & -0.044 & -0.047 \\
-0.047 & -0.044 & -0.029 & 0.027 & 0.238 & 0.027 & -0.029 & -0.044 \\
-0.044 & -0.047 & -0.044 & -0.029 & 0.027 & 0.238 & 0.027 & -0.029 \\
-0.029 & -0.044 & -0.047 & -0.044 & -0.029 & 0.027 & 0.238 & 0.027 \\
0.027 & -0.029 & -0.044 & -0.047 & -0.044 & -0.029 & 0.027 & 0.238
\end{array}\right] \text { La suma }
$$

en cada hilera ( $\mathrm{r}$ ) o columna (c) es: $\sum_{\mathrm{r}=0}^{\mathrm{p}-1} \mathrm{a}^{\mathrm{r}}=\sum_{\mathrm{c}=0}^{\mathrm{p}-1} \mathrm{a}^{\mathrm{c}}=\frac{1}{2 \mathrm{~s}}=\frac{1}{2(5)}=0.1$ 
Cuando sólo se dispone de una calculadora de escritorio podrían aplicarse las metodologías de Kempthorne y Curnow (1961); Singh y Chaudhary (1985); Martínez (1991). Para obtener los elementos de la primera hilera de su inversa Martínez (1991) calculó sus raíces características cómo: $\lambda \mathrm{j}=\sum_{l=1}^{p} b_{1 l} \operatorname{Cos}\left\{j\left[\frac{2 \pi(l-1)}{p}\right]\right\}, \mathrm{j}=1,2,3, \ldots, \mathrm{p}$. En ésta, $\lambda \mathrm{p}=\sum_{l=1}^{p} b_{1 l}=2 s$. $\lambda_{1}=$ $b_{11} \cos \left(\frac{2 \pi(1-1)}{8}\right)+b_{12} \cos \left(\frac{2 \pi(2-1)}{8}\right)+b_{13} \cos \left(\frac{2 \pi(3-1)}{8}\right)+b_{14} \cos \left(\frac{2 \pi(4-1)}{8}\right)+b_{15} \cos \left(\frac{2 \pi(5-1)}{8}\right)+$ $b_{16} \cos \left(\frac{2 \pi(6-1)}{8}\right)+b_{17} \cos \left(\frac{2 \pi(7-1)}{8}\right)+b_{18} \cos \left(\frac{2 \pi(8-1)}{8}\right)$

Las cruzas $1 \mathrm{x} 2$ y $1 \mathrm{x} 8$ no fueron muestreadas $\left(\mathrm{b}_{12}\right.$ y $\left.\mathrm{b}_{18}=0\right)$ y serán eliminadas; en $\mathrm{b}_{11}$ su coeficiente es $\mathrm{s}=5 \mathrm{y}$ en $\mathrm{b}$ 's restantes es 1 , después de reagrupar sus componentes: $\lambda_{1}=5 \cos (0)+$ $\cos \left(\frac{\pi}{2}\right)+\cos \left(\frac{3 \pi}{4}\right)+\cos (\pi)+\cos \left(\frac{5 \pi}{4}\right)+=5-0.00000367-0.7071-1-0.7071+0.000011=$ 2.5858. $\lambda_{2}=5 \cos (0)+\cos (\pi)+\cos \left(\frac{3 \pi}{2}\right)+\cos (2 \pi)+\cos \left(\frac{5 \pi}{2}\right)+\cos (3 \pi)=5-1+0.000011$ $+1-0.0000183-1=4$. Similarmente: $\lambda_{3}=5.4141 ; \lambda_{4}=6 ; \lambda_{5}=5.4140 ; \lambda_{6}=4 ; \lambda_{7}=2.5857 ; \lambda_{8}=2 \mathrm{~s}=$ 10 .

Kempthorne y Curnow (1961) estimaron las $\lambda$ 's cómo: $\lambda_{\mathrm{j}}=s-\frac{\sin (n-2) \frac{j \pi}{n}}{\sin \frac{j \pi}{n}}=\frac{s\left[\sin \left(\frac{j \pi}{n}\right)\right]-\operatorname{Sin}(n-s)\left(\frac{j \pi}{n}\right)}{\operatorname{Sin}\left(\frac{j \pi}{n}\right)}$. Dónde: $\mathrm{j}=1,2, \ldots, \mathrm{n}-1 ; \lambda_{\mathrm{n}}=2 \mathrm{~s}, \mathrm{y}$ s es el número de cruzas muestreadas por progenitor. $\lambda_{1}=$ $\frac{5\left[\operatorname{Sin}\left(\frac{\pi}{8}\right)\right]-\operatorname{Sin}(8-5)\left(\frac{\pi}{8}\right)}{\operatorname{Sin}\left(\frac{\pi}{8}\right)}=\quad \frac{5[\operatorname{Sin}(0.3927)]-\operatorname{Sin}(1.1781)}{\operatorname{Sin}(0.3927)}=\quad 2.5857 . \quad \lambda_{2}=\quad \frac{5\left[\operatorname{Sin}\left(\frac{2 \pi}{8}\right)\right]-\operatorname{Sin}(8-5)\left(\frac{2 \pi}{8}\right)}{\operatorname{Sin}\left(\frac{2 \pi}{8}\right)}=$ $\frac{5[\operatorname{Sin}(0.7854)]-\operatorname{Sin}(2.3562)}{\operatorname{Sin}(0.7854)}=4$. Las $\lambda$ 's restantes son idénticas a las calculadas con el método de Martínez (1991). Con estas se obtienen los elementos de la primera hilera de la inversa de la matriz A. $\mathrm{a}^{\mathrm{o}}=\left(\frac{1}{n}\right) \llbracket\left(\frac{1}{\lambda 1}+\frac{1}{\lambda 2}+, \ldots,+\frac{1}{\lambda 8}\right)=\left(\frac{1}{8}\right) \llbracket\left(\frac{1}{2.5857}+\frac{1}{4}+\frac{1}{5.4142}+\frac{1}{6}+\frac{1}{5.4142}+\frac{1}{4}+\frac{1}{2.5857}+\frac{1}{10}\right)=0.2387$. Los otros elementos de la primera hilera se calculan así: $\mathrm{a}^{\mathrm{j}}=\left(\frac{1}{n}\right) \sum_{l=1}^{n} \frac{1}{\lambda_{l}} \operatorname{Cos} \frac{j(n-l)}{n}(2 \pi)$. En ésta, $\mathrm{j}=1$, $2, \ldots, \quad \mathrm{n}-1 . \mathrm{a}^{1}=\left(\frac{1}{8}\right)\left[\frac{1}{\lambda 1} \operatorname{Cos} \frac{1(8-1)(2 \pi)}{8}+\frac{1}{\lambda 2} \operatorname{Cos} \frac{1(8-2)(2 \pi)}{8}+\frac{1}{\lambda 3} \operatorname{Cos} \frac{1(8-3)(2 \pi)}{8}+\frac{1}{\lambda 4} \operatorname{Cos} \frac{1(8-4)(2 \pi)}{8}+\right.$ $\frac{1}{\lambda 5} \operatorname{Cos} \frac{1(8-5)(2 \pi)}{8}+\frac{1}{\lambda 6} \operatorname{Cos} \frac{1(8-6)(2 \pi)}{8}+\frac{1}{\lambda 7} \operatorname{Cos} \frac{1(8-7)(2 \pi)}{8}+\frac{1}{\lambda 8} \operatorname{Cos} \frac{1(8-8)(2 \pi)}{8}=\left(\frac{1}{8}\right)\left[\frac{1}{\lambda 1} \operatorname{Cos} \frac{7 \pi}{4}+\right.$ $\frac{1}{\lambda 2} \operatorname{Cos} \frac{(3 \pi)}{2}+\frac{1}{\lambda 3} \operatorname{Cos} \frac{(5 \pi)}{4}+\frac{1}{\lambda 4} \operatorname{Cos}(\pi)+\frac{1}{\lambda 5} \operatorname{Cos} \frac{(3 \pi)}{4}+\frac{1}{\lambda 6} \operatorname{Cos} \frac{(\pi)}{2}+\frac{1}{\lambda 7} \operatorname{Cos} \frac{(\pi)}{4}+\frac{1}{\lambda 8} \operatorname{Cos}(0)=$ $\left(\frac{1}{8}\right)(0.2734+0.00000275-0.1306-0.1666666-0.1306-0.00000092+0.27347+0.1=$ $\frac{0.219}{8}=0.027$. Este es el valor de la hilera 1, columna 2 .

Los otros elementos se calculan similarmente, como la matriz es simétrica y circulante, para obtener los de la siguiente hilera simplemente se desplazan los de la anterior una columna a la derecha. Este procedimiento se repite hasta completar todas las hileras en la inversa de la matriz.

\section{Cálculo de la suma corregida de las cruzas muestreadas $\left(Q_{i}\right)$}

El factor de corrección (FCQ) se calcula con las mismas restricciones que para SC cruzas: FCQ = $\frac{2 \sum_{\mathrm{i}=1}^{\mathrm{p}} \sum_{\mathrm{j}=1}^{\mathrm{p}} \overline{\mathrm{Y}}_{\mathrm{ij}}}{\mathrm{ps}}=\frac{2(135.877)}{8(5)}=\frac{\frac{2 \sum_{\mathrm{i}=1}^{\mathrm{t}} \sum_{\mathrm{j}=1}^{\mathrm{p}} \mathrm{Y}_{\mathrm{ij}}}{\mathrm{r}}}{\mathrm{ps}}=\frac{\frac{2 \mathrm{Y} \ldots}{\mathrm{r}}}{\mathrm{ps}}=\frac{\frac{2(543.53)}{4}}{8(5)}=6.794 \mathrm{t} \mathrm{ha}^{-1}$. Las medias corregidas de las 20 cruzas se calculan como la diferencia entre el promedio aritmético de cada una de ellas y FCQ. Para la cruza 1x3=7.292 - 6.794=0.498. Los valores restantes se muestran en el (Cuadro 4). 
Cuadro 4. Medias aritméticas sin corregir o corregidas (arriba o abajo de la diagonal).

\begin{tabular}{ccccccccc}
\hline \multirow{2}{*}{ Línea i $($ ( ) $)$} & \multicolumn{8}{c}{ Línea j $($ đ $)$} \\
\cline { 2 - 9 } & P1 & P2 & P3 & P4 & P5 & P6 & P7 & P8 \\
\hline P1 & - & - & 7.292 & 6.995 & 6.625 & 6.862 & 6.077 & - \\
P2 & - & - & - & 6.432 & 7.042 & 7.762 & 6.917 & 7.857 \\
P3 & 0.498 & - & - & - & 6.427 & 7.24 & 6.33 & 6.82 \\
P4 & 0.201 & -0.361 & - & - & - & 5.845 & 6.535 & 7.105 \\
P5 & -0.168 & 0.248 & -0.366 & - & - & - & 5.942 & 7.152 \\
P6 & 0.068 & 0.968 & 0.446 & -0.948 & - & - & - & 6.62 \\
P7 & -0.716 & 0.123 & -0.463 & -0.258 & -0.851 & - & - & - \\
P8 & - & 1.063 & 0.026 & 0.311 & 0.358 & -0.173 & - & - \\
\hline
\end{tabular}

La suma de los $\mathrm{Q}_{\mathrm{i}}$ es cero y éstos se calculan cómo: $\mathrm{Q}_{1}=1 \times 3+1 \times 4+1 \times 5+1 \times 6+1 \times 7=0.498+$ $0.201-0.168+0.068-0.716=-0.118 . \mathrm{Q}_{2}=2 \times 4+2 \times 5+2 \times 6+2 \times 7+2 \times 8=-0.361+0.248+$ $0.968+0.123+1.063=2.041$. También: $\mathrm{Q}_{3}=0.140 ; \mathrm{Q}_{4}=-1.057 ; \mathrm{Q}_{5}=-0.781 ; \mathrm{Q}_{6}=0.360 ; \mathrm{Q}_{7}=-$ $2.168 ; \mathrm{Q}_{8}=1.585$.

\section{Estimación de los efectos de aptitud combinatoria general (ACG)}

La estimación de la ACG para cada progenitor $\left(\mathrm{g}_{\mathrm{i}}\right)$ se hace con álgebra de matrices. $\underline{\mathrm{A}} \underline{\mathrm{G}}=\underline{\mathrm{H}}$ su solución es: $\mathrm{G}=\mathrm{A}^{-1} \mathrm{H}$. Dónde: $\mathrm{A}^{-1}$ es la inversa de la matriz $\mathrm{A} ; \mathrm{H}$ es un vector columna formado por los valores de las sumas corregidas de las cruzas muestreadas $\left(\mathrm{Q}_{\mathrm{i}}\right)$, y $\mathrm{G}$ es otro vector columna integrado por las estimaciones de $\mathrm{g}_{\mathrm{i}}$.

\begin{tabular}{|c|c|c|c|c|c|c|c|c|c|}
\hline \multicolumn{10}{|c|}{ Así: $\mathrm{G}_{8 \times 1}=\mathrm{A}^{-1}{ }_{8 \times 8} \mathrm{H}_{8 \times 1}$} \\
\hline [ 0.238 & 0.027 & -0.029 & -0.044 & -0.047 & -0.044 & -0.029 & 0.027 & {$[-0.118]$} & $0.1977\rceil$ \\
\hline 0.027 & 0.238 & 0.027 & -0.029 & -0.044 & -0.047 & -0.044 & -0.029 & 2.041 & 0.5853 \\
\hline-0.029 & 0.027 & 0.238 & 0.027 & -0.029 & -0.044 & -0.047 & -0.044 & 0.140 & 0.1028 \\
\hline-0.044 & -0.029 & 0.027 & 0.238 & 0.027 & -0.029 & -0.044 & -0.047 & -1.057 & -0.3137 \\
\hline-0.047 & -0.044 & -0.029 & 0.027 & 0.238 & 0.027 & -0.029 & -0.044 & -0.781 & -0.3005 \\
\hline-0.044 & -0.047 & -0.044 & -0.029 & 0.027 & 0.238 & 0.027 & -0.029 & 0.360 & -0.1071 \\
\hline-0.029 & -0.044 & -0.047 & -0.044 & -0.029 & 0.027 & 0.238 & 0.027 & -2.168 & -0.4879 \\
\hline 0.027 & -0.029 & -0.044 & -0.047 & -0.044 & -0.029 & 0.027 & 0.238 & $1.585\rfloor$ & 0.3236 \\
\hline
\end{tabular}

El valor de $g_{1}$ se obtiene como la sumatoria de los productos de la primera hilera de la inversa de la matriz $\underline{\mathrm{A}}$ con la única columna de la matriz $\underline{\mathrm{H}}$; es decir: $\mathrm{g}_{1}=[(0.238)(-0.118)+(0.027)(2.041)$ $+(-0.029)(0.114)+, \ldots,+(0.027)(1.585)]=0.1977$. También: $g_{2}=0.5853 ; g_{3}=0.1028 ; g_{4}=-0.3137$; $\mathrm{g}_{5}=-0.3005 ; \mathrm{g}_{6}=-0.1071 ; \mathrm{g}_{7}=-0.4879 ; \mathrm{g}_{8}=0.3236$.

\section{Estimación de valores $S_{i j}$}

Los efectos de aptitud combinatoria específica $\left(S_{\mathrm{ij}}\right)$ para cada cruza simple se estiman cómo: $\mathrm{S}_{\mathrm{ij}}=$

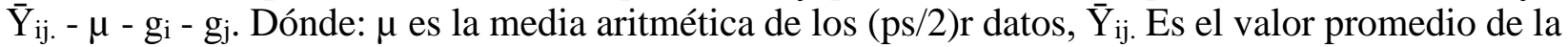
cruza entre los progenitores $\mathrm{i}, \mathrm{j}, \mathrm{g}_{\mathrm{i}}$, $\mathrm{g}_{\mathrm{j}}$ son las estimaciones de aptitud combinatoria general de las líneas $\mathrm{i}, \mathrm{j}$. Las restricciones son: $\sum_{\mathrm{i}=1}^{\mathrm{p}} \mathrm{g}_{\mathrm{i}}=0 \mathrm{y} \sum_{\mathrm{j}=1}^{\mathrm{p}-1} \mathrm{~S}_{\mathrm{ij}}=0$. Para las cruzas que involucran al progenitor 1, se tendrá: $S_{13}=\bar{Y}_{13}-\mu-g_{1}-g_{3}=7.292-6.794-0.1977-0.1028=0.1974$. Similarmente: $S_{14}=$ $0.3172 ; \mathrm{S}_{15}=-0.066 ; \mathrm{S}_{16}=-0.0223 ; \mathrm{S}_{17}=-0.4265$. 


\section{Cálculo de la SC ACG}

La suma de cuadrados entre tratamientos se divide en ACG y ACE; si se conoce el valor de dos de estas tres fuentes de variación en la tabla dialélica, la tercera se calcula por diferencia. $\mathrm{SC}$ ACG= $\mathrm{r} \Sigma \underline{\mathrm{G}}_{\mathrm{i}}{ }^{\prime} \underline{\text { Hi}}$. Dónde: $\mathrm{r}=$ número de repeticiones, $\mathrm{G}_{\mathrm{i}}{ }^{\prime}=$ transpuesta del vector columna $8 \mathrm{x} 1$ formada por los valores de $\mathrm{g}_{\mathrm{i}}$ y $\mathrm{H}_{\mathrm{i}}=$ vector columna integrado con los valores de $\mathrm{Q}_{\mathrm{i}}$, se multiplica por $\mathrm{r}$ porqué se utilizaron promedios aritméticos. SC ACG $=4[(0.1977)(-0.118)+(0.5853)(2.041)+$ $(0.3236)(1.585)=13.137 . \mathrm{SC}$ ACE $=\mathrm{SC}$ Cruzas-SC ACG $=22.439-13.137=9.3019$.

Del Cuadro 5 puede concluirse que sólo entre repeticiones y entre efectos de aptitud combinatoria general hubo diferencias significativas ( $p=0.05 \mathrm{y} p=0.01$, respectivamente). Estos resultados indican que el bloqueo en el área experimental, establecido en sentido perpendicular al gradiente de heterogeneidad ambiental, fue eficiente. Esto contribuyó a disminuir el error experimental. Con relación al rendimiento de grano de las 20 cruzas muestreadas, en éstas los efectos genéticos aditivos fueron de mayor importancia que los no aditivos (dominancia y epistasis), por lo que este grupo de ocho progenitores de maíz podría utilizarse eficientemente en la formación de variedades sintéticas. En las generaciones segregantes $\left(\mathrm{F}_{2} \mathrm{O}\right.$ superior), provenientes del apareamiento aleatorio entre este grupo de líneas endogámicas, podrían seleccionarse plantas destinadas a la obtención de nuevas y mejores variedades de polinización libre.

Cuadro 5. Anava con partición de efectos entre cruzas en aptitud combinatoria.

\begin{tabular}{|c|c|c|c|c|c|c|}
\hline \multirow{3}{*}{$\begin{array}{l}\text { Fuente de } \\
\text { variación }\end{array}$} & \multirow{3}{*}{$\begin{array}{c}\text { Grados de } \\
\text { libertad }\end{array}$} & \multirow{3}{*}{$\begin{array}{l}\text { Suma de } \\
\text { cuadrados }\end{array}$} & \multirow{3}{*}{$\begin{array}{c}\text { Cuadrados } \\
\text { medios }\end{array}$} & \multicolumn{3}{|c|}{ Valores de F } \\
\hline & & & & \multirow{2}{*}{ Calculado } & \multicolumn{2}{|c|}{ Tablas } \\
\hline & & & & & $5 \%$ & $1 \%$ \\
\hline Repeticiones & 3 & 17.07 & 5.69 & $6.84^{* * *}$ & 2.78 & 4.16 \\
\hline Cruzas & (19) & $(22.43)$ & 1.18 & $1.42 \mathrm{~ns}$ & 1.75 & 2.22 \\
\hline $\mathrm{ACG}$ & 7 & 13.137 & 1.876 & $2.25^{*}$ & 2.18 & 2.98 \\
\hline $\mathrm{ACE}$ & 12 & 9.302 & 0.775 & $0.93 \mathrm{~ns}$ & 1.93 & 2.53 \\
\hline Error & 57 & 47.38 & 0.83 & & & \\
\hline Total & 79 & 86.89 & & & & \\
\hline
\end{tabular}

GL ACG = p-1= 8-1=7; GL ACE= GL cruzas-GL ACG= 19-7=12. También, GL ACE= $[\mathrm{p}(\mathrm{s}-2) / 2]=[8(5-2) / 2]=12$.

Estimación de componentes de varianza y heredabilidad. $\sigma^{2}=$ cuadrado medio del error $=0.8312$. $\sigma^{2}{ }_{s}=(\mathrm{CM}$ de ACE - CM del error $) / r=(0.7751-0.8312) / 4=-0.014 . \sigma^{2} \mathrm{~g}=(\mathrm{p}-1)(\mathrm{CM}$ ACG $-\mathrm{CM}$ $\mathrm{ACE}) / \mathrm{rs}(\mathrm{p}-2)=7(1.8767-0.7751) / 4(5)(6)=0.0642$. La varianza promedio para calcular las diferencias entre dos valores de $g_{i}$ estimados se calcula cómo: VM $\left(g_{i}-g_{j}\right)=2\left\{\frac{p a^{\circ}}{p-1}-\right.$ $\left.\frac{1}{2 \mathrm{~s}(\mathrm{p}-1)}\right\}\left(\sigma_{\mathrm{s}}^{2}+\frac{\sigma_{\mathrm{e}}^{2}}{\mathrm{r}}\right)=2\left\{\frac{8(0.238)}{7}-\frac{1}{2(5)(7)}\right\}\left(-0.014+\frac{0.8312}{4}\right)=2(0.272-0.014286)(0.1938)=0.0998$. Y su error estándar es: $\mathrm{EE}\left(\mathrm{g}_{\mathrm{i}}-\mathrm{g}_{\mathrm{j}}\right)=\sqrt{\mathrm{VM}\left(\mathrm{g}_{\mathrm{i}}-\mathrm{g}_{\mathrm{j}}\right)}=\sqrt{0.0998}=0.3159$.

La equivalencia entre las varianzas calculadas previamente y las aditivas y de dominancia se establece cuando se asume que el coeficiente de endogamia es igual a uno, debido a que las líneas usadas en el dialélico parcial son endogámicas $\left(S_{7}\right)$. Entonces: $\sigma_{\mathrm{A}}^{2}=2 \sigma_{\mathrm{g}}^{2}=2(0.0642)=0.1284$; 
$\sigma^{2}{ }_{D}=\sigma^{2}{ }_{S}=-0.014$. La varianza genética total, $\sigma^{2}$, se estima cómo: $\sigma^{2}{ }_{G}=\sigma^{2}{ }_{A}+\sigma_{D}^{2}=0.1284-0.014$ $=0.1144$. La heredabilidad en sentido amplio, $\mathrm{H}^{2}$, se estima cómo: $\mathrm{H}^{2}=100\left(\sigma_{\mathrm{G}}^{2} / \sigma^{2} \mathrm{~F}\right)=100$ $(0.1144 / 0.3222)=35.5 \%$, donde $\sigma^{2}$ es la varianza fenotípica. $\sigma^{2}{ }_{F}=2 \sigma_{g}^{2}+\sigma^{2}{ }_{s}+\left(\sigma^{2}{ }_{e} / r\right)=0.1284-$ $0.014+0.2078=0.3222$. La heredabilidad en sentido estrecho, $\mathrm{h}^{2}$, se estima cómo: $\mathrm{h}^{2}=100$ $\left(\sigma^{2}{ }_{\mathrm{A}} / \sigma_{\mathrm{F}}^{2}\right)=100(0.1284 / 0.3222)=39.85 \%$.

La estimación negativa de la varianza de aptitud combinatoria específica (efectos genéticos no aditivos) sugiere que hubo una subestimación en la heredabilidad en sentido amplio (35.5\%) comparativo con la estimación de la heredabilidad en sentido estrecho (39.85\%). El valor de $\mathrm{h}^{2}$ sugiere que la variabilidad fenotípica total medida en las 20 cruzas simples de maíz está relacionada con genes aditivos que determinan el rendimiento de grano. En este contexto se asume que 39.8\% de la variabilidad fenotípica total estimada en esta variable cuantitativa se atribuye a las diferencias que existen entre los progenitores endogámicos, cuyos efectos predominantes son aditivos.

\section{Resultados obtenidos con OPSTAT}

Character 1. Analysis of variance table for RBD.

\begin{tabular}{cccccc}
\hline Source of Variation & DF & Sum of Squares & Mean Squares & F-Calculated & Signficance \\
\hline Replications & 3 & 17.074 & & & \\
Treatments & 19 & 22.438 & 1.181 & 1.421 & 0.15415 \\
Error & 57 & 47.385 & 0.831 & & \\
Total & 79 & 86.897 & & & \\
\hline
\end{tabular}

Mean and standard error table

\begin{tabular}{|c|c|c|}
\hline Cross & Mean & Standard error \\
\hline $1 \times 3$ & 7.293 & 1.665 \\
\hline $1 \mathrm{X} 4$ & 6.995 & 0.272 \\
\hline $1 \times 5$ & 6.625 & 0.48 \\
\hline $1 \times 6$ & 6.863 & 1.191 \\
\hline $1 \times 7$ & 6.077 & 0.552 \\
\hline $2 \times 4$ & 6.433 & 0.193 \\
\hline $2 \times 5$ & 7.043 & 1.001 \\
\hline $2 \times 6$ & 7.763 & 0.774 \\
\hline $2 \times 7$ & 6.918 & 0.187 \\
\hline $2 \times 8$ & 7.858 & 0.925 \\
\hline $3 \times 5$ & 6.428 & 0.494 \\
\hline $3 \times 6$ & 7.24 & 1.468 \\
\hline $3 \times 7$ & 6.33 & 0.32 \\
\hline $3 \times 8$ & 6.82 & 1.033 \\
\hline $4 \times 6$ & 5.845 & 1.378 \\
\hline $4 \times 7$ & 6.535 & 0.317 \\
\hline $4 \times 8$ & 7.105 & 1.127 \\
\hline
\end{tabular}




\begin{tabular}{lcc}
\hline Cross & Mean & Standard error \\
\hline $5 \times 7$ & 5.943 & 0.23 \\
$5 \times 8$ & 7.153 & 0.927 \\
$6 \times 8$ & 6.62 & 1.018 \\
\hline
\end{tabular}

Los errores estándar de la tabla anterior corresponden a $\sqrt{\sigma_{i j}^{2}}$

GCA effect are

\begin{tabular}{llllllll}
\hline 0.198 & 0.586 & 0.103 & -0.314 & -0.300 & -0.107 & -0.488 & 0.324 \\
\hline
\end{tabular}

Combining ability analysis

\begin{tabular}{cccccc}
\hline Source of variation & DF & Sum of squares & Mean squares & F-calculated & Signficance \\
\hline Replications & 3 & 17.074 & 5.691 & & \\
Crosses & 19 & 22.438 & 1.181 & 1.421 & 0.15415 \\
Due to GCA & 7 & 13.142 & 1.877 & $2.258^{*}$ & 0.0423 \\
Due to SCA & 12 & 9.296 & 0.775 & 0.932 & 0.52237 \\
Error & 57 & 47.385 & 0.831 & & \\
Total & 79 & 86.897 & & & \\
\hline
\end{tabular}

Estimation of component of variances.

$\begin{array}{cc}\text { SIGMASQ-G } & 0.064 \\ \text { SIGMASQ-S } & -0.014 \\ \text { SIGMASQ-A } & 0.129 \\ \text { SIGMASQ-D } & -0.014 \\ \text { Average variance(gi-gj) } & 0.100 \\ \text { SE (gi-gj) (EE) } & 0.316\end{array}$

La equivalencia entre las varianzas reportadas en el cuadro anterior, con las de la sección de estimación de heredabilidades, es $\sigma_{\mathrm{g}}^{2}, \sigma_{\mathrm{s}}^{2}, \sigma_{\mathrm{A}}^{2}, \sigma_{\mathrm{D}}^{2}, \mathrm{VM}$, respectivamente. Heritability (Narrow Sense) $=1.12$.

\section{Conclusiones}

La obtención de la inversa de la matriz A es muy laboriosa cuando se utiliza una calculadora de escritorio, pero la estimación de sus raíces características, $\lambda$ 's, se simplifica cuando se aplica el método de Kempthorne y Curnow (1961), en comparación con el de Martínez (1991) y ambos conducen a resultados similares; con éstas, también es más fácil estimar los elementos de su primera hilera. Como la matriz $\mathrm{A}^{-1}$ es simétrica y circulante, los elementos de la segunda hilera se obtienen desplazando los de la primera a la siguiente columna y así sucesivamente. Si no es posible el uso de software, ambas metodologías serán de gran utilidad. Adicionalmente, se observó que hay pocos paquetes estadísticos para el análisis de un dialélico incompleto que estén disponibles gratuitamente. 
El software Opstat analiza experimentos en un solo ambiente, éste verificó fácil, rápida y confiablemente los análisis obtenidos con la calculadora de escritorio, incluidos la varianza promedio y los errores estándar para cualquier contraste de $\mathrm{g}_{\mathrm{i}}$ con $\mathrm{g}_{\mathrm{j}}$. Si se ignora $\mathrm{S}_{\mathrm{ij}}$ en el modelo genético, se puede estimar el potencial de producción de las cruzas simples usando los dos métodos de Kempthorne y Curnow (1961). Cómo la varianza de dominancia fue negativa el software estimó $\mathrm{h}^{2}$ incorrectamente.

\section{Literatura citada}

Awata, L. A. O.; Tongoona, P.; Danquah, E.; Efie, B. E. and Marchelo-Dragga, P. W. 2018. Common mating designs in agricultural research and their reliability in estimation of genetic parameters. IOSR J. Agric. Vet. Sci. 11(7):16-36.

Christie, B. R. and Shattuck, V. I. 1992. The diallel cross: design, analysis, and use for plant breeders. Plant Breed. Reviews. 9(1):19-36.

Chaudhary, B. D.; Kakar, S. N. and Singh, R. K. 1977. Comparison of diallel and its modifications. Silvae genetica. 26(1):61-120.

Fyfe, J. L. and Gilbert, N. 1963. Partial diallel crosses. Biometrics. 19(2):278-286.

Griffing, B. 1956. Concept of general and specific combining ability in relation to diallel crossing systems. Aust. J. Biol. Sci. 9(4):463-493.

Gomez, K. A. and Gomez, A. A. 1984. Statistical procedures for agricultural research. $2^{\text {nd }}$ (Ed.). John Wiley and Sons, Inc. Singapore. 690 p.

González, H. A.; Sahagún, C. J. y Pérez, L. D. J. 2007. Estudio de ocho líneas de maíz en un experimento dialélico incompleto. Rev. Cienc. Agríc. Inf. 16(1):3-9.

Hinkelmann, K. and Stern, K. 1960. Kreuzungspläne zur selektionszüchtung bei waldbäumen. Silvae genetica. 9(3):121-133.

Kempthorne, O. and Curnow, R. N. 1961. The partial diallel cross. Biometrics. 17(2):229-250.

Martínez, G. A. 1991. Análisis de los experimentos dialélicos a través del procedimiento IML de SAS. Colegio de Postgraduados, Chapingo, Estado de México. Comunicaciones en estadística y cómputo. Centro de estadística y cálculo. 10(2):1-36.

Martínez, G. A. 1998. Diseños experimentales: métodos y elementos de teoría. (Ed.). Trillas, México, DF. 756 p.

Miranda F. J. B. and Vencovsky, R. 1999. The partial circulant diallel cross at the interpopulation level. Gen. Mol. Biol. 22:249-255.

Muntaz, A.; Zafar, F. and Aamar, S. S. 2015. A review on mating designs. Nat. Sci.13(2):98-105.

Murthy, B. R.; Arunachalam, V. and Anand, I. J. 1966. Diallel and partial diallel analysis of some yield factors in Linum usitatissimum. Heredity. 22(1):35-41.

Pérez, L. D. J.; Franco, M. J. R. P.; Gutiérrez, R. F.; Hernández, A. J.; Balbuena, M. A. y González, H. A. 2021. Diseño de experimentos factoriales $2^{\mathrm{n}}$ para su análisis con infoStat e infoGen. Rev. Mex. Cienc. Agríc. 12(6):1087-1099.

Rojas, M. B. A. 1973. Design and analysis of diallel crosses. Department of experimental statistics. New Mexico state university. $14 \mathrm{p}$.

Saavedra, G. C.; Pérez, L. D. J.; González, H. A.; Franco, M. J. R. P.; Rubí, A. M. y Ramírez, D. J. F. 2021. Métodos de Griffing: revisión sobre su importancia y aplicación en fitomejoramiento convencional. Rev. Mex. Cienc. Agríc. 12(7):1275-1286. 
Shunmugathai, R. and Srinivasan, M. R. 2012. Robustness of PDC Plan using BIB mating designs against unavailability of one or more observations. Inter. J. Mathematics Sci. Comp. 2(2):32-40.

Silva, C. M.; Miranda, F. J. B.; Mendes, U. C. and Reis, E. F. 2017. Partial diallel cross for predicting yield of semiexotic maize populations. Gen. Mol. Res. 16(1): 1-16.

Singh, R. K. and Chaudhary, B. D. 1985. Biometrical methods in quantitative genetic analysis. Revised (Ed.). Kalyani publishers. New Delhi-Ludhiana, printed in India. 318 p. 